DR. ANNALISA BERZIGOTTI (Orcid ID : 0000-0003-4562-9016)

Article type : Original Articles

Editor : Luca Valenti

\title{
Controlled Attenuation Parameter reflects steatosis in compensated advanced chronic liver disease
}

Rosangela Piccinni ${ }^{1}$, Susana G. Rodrigues ${ }^{1}$, Matteo Montani ${ }^{2}$, Giuseppe Murgia ${ }^{1}$, Maria Gabriela Delgado ${ }^{1}$, Stefania Casu ${ }^{1}$, Guido Stirnimann ${ }^{1}$, Nasser Semmo ${ }^{1}$, Andrea De Gottardi $^{1}$, Jean-François Dufour ${ }^{1}$, Annalisa Berzigotti ${ }^{1}$

1 University Clinic for Visceral Surgery and Medicine (UVCM), Inselspital, University of Bern, Switzerland; 'Institute of Pathology, University of Bern, Switzerland

\section{Correspondence:}

Annalisa Berzigotti, MD, PhD

Swiss Liver Center, Hepatology, University Clinic for Visceral Surgery and Medicine, Inselspital, University of Bern

MEM F807, Murtenstrasse 35, CH - 3010 Bern, Switzerland

Tel. +4131632 87 27; Fax. +413163249 97

E-mail: annalisa.berzigotti@insel.ch

Main body word count: 2802

Total number figures and tables: 7: 3 main tables, 1 supplementary table, 3 figures

\section{Abbreviations}

AUROC- area under the receiving operating characteristic curves; BMI- Body Mass Index; cACLD- compensated advanced chronic liver disease; CAP- Controlled Attenuation Parameter; HVPG- hepatic venous pressure gradient; IQR- interquartile range; IQR/M- IQR/Median; LR- likelihood ratio; LSM- liver stiffness measurement; NAFLD- non alcoholic fatty liver disease; NASH- non alcoholic steatohepatitis; TJLBtransjugular liver biopsy; ULN- upper limit of normality

This article has been accepted for publication and undergone full peer review but has not been through the copyediting, typesetting, pagination and proofreading process, which may lead to differences between this version and the Version of Record. Please cite this article as doi: $\underline{10.1111 / \text { LIV.14325 }}$

This article is protected by copyright. All rights reserved 


\section{Statement of author contributions:}

RP: design of the database; acquisition of data; analysis of the data and drafting the manuscript; SGR, MM, GM: acquisition of data, interpretation of data and manuscript critical revision for important intellectual content; MGD, SC, NS, GS, ADG, JFD: manuscript critical revision for important intellectual content; $A B$ : study concept and design, analysis and interpretation of data, drafting of manuscript; study supervision. All authors have read and approved the final version.

\section{Disclosures:}

The authors have no potential conflicts (financial, professional, or personal) that are relevant to the manuscript to disclose.

\section{Acknowledgements:}

Susana G. Rodrigues receives financial support from the Stiftung für Leberkrankheiten Bern. Annalisa Berzigotti received an Interdisciplinary Grant 2015 of the University of Bern (UniBe-ID 2015). 


\section{Abstract}

Background \& Aims: Controlled Attenuation Parameter (CAP) for steatosis assessment has not been validated in compensated advanced chronic liver disease cACLD. We primarily aimed at assessing the accuracy of CAP for the diagnosis and quantification of steatosis in CACLD. Secondary aim: to assess the validity of non-invasive criteria for cACLD according to liver stiffness measurement (LSM).

Methods: This is a single center retrospective study including patients with cACLD defined as LSM $\geq 10 \mathrm{kPa}$, CAP measurement and liver biopsy (reference standard for steatosis and fibrosis) observed in 06/2015-06/2017. Steatosis was graded as S0 (<5\%), S1 (5-32\%), S2 (33-66\%) and S3 (>66\%). The diagnostic performance of CAP for any grade of steatosis and for high-grade steatosis $(\geq \mathrm{S} 2)$ was studied.

Results: Among 461 consecutive patients, 111 with LSM-based diagnosis of CACLD were included $\left(63 \%\right.$ male, median age $55 \mathrm{yrs}$, median BMI $28.1 \mathrm{Kg} / \mathrm{m}^{2}$, etiology: 32\% NAFLD/NASH, 32\% alcohol or viral +metabolic syndrome, 15\% viral, $6 \%$ autoimmune, $4 \%$ alcohol, $11 \%$ others). Median LSM and CAP were $16.1 \mathrm{kPa}$ and $277 \mathrm{~dB} / \mathrm{m}$, respectively. On liver biopsy, steatosis was found in 88/111 patients (79\%); 44 patients (43 with metabolic syndrome) had high-grade steatosis.

CAP was accurate in identifying any grade of steatosis (AUROC $0.847 ; 95 \% \mathrm{Cl}$ 0.767$0.926, \mathrm{p}<0.0001)$, and $\geq S 2$ steatosis $(0.860 ; 95 \% \mathrm{Cl} 0.788-0.932, \mathrm{p}<0.0001)$. CAP performed similarly in patients with CAP-IQR $\geq$ or $<40 \mathrm{~dB} / \mathrm{m}$.

Conclusions: Steatosis is frequent in patients with CACLD and metabolic syndrome. CAP diagnostic accuracy for any steatosis and high-grade steatosis is good in this population. A CAP-IQR $\geq 40 \mathrm{~dB} / \mathrm{m}$ does not impair CAP diagnostic accuracy in CACLD.

Keywords: Liver cirrhosis; steatosis, liver biopsy; liver stiffness; NASH. 


\section{Lay Summary}

- Steatosis (fatty liver) is currently frequent in patients with histologically confirmed advanced liver fibrosis or cirrhosis, particularly if features of metabolic syndrome ar present (obesity, increased arterial blood pressure, diabetes, increased cholesterol and/or triglycerids)

- CAP reflects intrahepatic fat content with good accuracy, and is more useful to exclude steatosis than to identify it 


\section{INTRODUCTION}

Steatosis due to nonalcoholic fatty liver disease (NAFLD) has become one of the most frequent causes of referral for chronic liver disease(1) and the progressive form of NAFLD (non-alcoholic steato-hepatitis-NASH) is associated with increased risk of cirrhosis(2) and hepatocellular carcinoma development, even in non-cirrhotic livers(3). Furthermore, steatosis is an important co-factor of progression in chronic liver disease of any cause $(4,5)$. In patients with compensated advanced chronic liver disease (cACLD, corresponding to bridging fibrosis or cirrhosis)(6), overweight/obesity- the main determinants of an increased liver fat content- predict clinical decompensation independent of portal pressure and serum albumin(7). Moreover, in patients with CACLD due to chronic Hepatitis $C$, steatosis was associated with a higher risk of progression to cirrhosis or its complications(8). This data underlines the importance of correctly diagnosing and quantifying liver fat content in patients with cACLD. However, liver biopsy is not routinely performed in patients with CACLD, who are well diagnosed by liver stiffness measurement (LSM). Recently, controlled attenuation parameter (CAP) has become available $(9,10)$. This simple, non-invasive technique allows diagnosing and quantifying liver fat content simultaneously to LSM using FibroScan®(11). A recent metaanalysis confirmed that CAP is a reliable method to diagnose significant steatosis(12). On the other hand, LSM are higher in patients with higher CAP, and, in turn, patients with LSM >10 kPa show higher CAP values even in the absence of severe steatosis, suggesting a complex interaction between the $\operatorname{two}(12)(13,14)$. Since severe fibrosis or cirrhosis was present in only $20-25 \%$ of cases included in previous studies $(12,15)$, CAP has been insufficiently studied in this population. In addition, it is assumed that liver fat content spontaneously decreases as liver fibrosis progresses to cirrhosis $(16,17)$, and high grades of steatosis are considered unlikely in CACLD. However, in a study performed by our group in patients with cACLD, CAP values $\geq 220 \mathrm{~dB} / \mathrm{m}$ were associated with clinically relevant events (18), but due to the lack of histological data, we could not prove that a higher CAP mirrored an increased fat content in this specific population. We hypothesized that due to the increased prevalence of obesity, liver steatosis would be frequent on histology in a contemporary population of patients with CACLD of any cause, and that CAP would maintain its diagnostic ability even in this specific group of patients. 
The primary aim of the present study was then to assess the accuracy of CAP for diagnosis and quantification of steatosis in patients with CACLD of any cause taking histology as a gold standard. Secondary aims were to assess the performance of the non-invasive criteria for CACLD suggested by the Baveno VI recommendations based on LSM and the reliability of CAP measurement based on CAP interquartile range (IQR) (13) in patients with cACLD. 


\section{METHODS}

This is a single center retrospective study that took place at Hepatology, Inselspital, University of Bern, Switzerland. The study was approved by the Ethical Committee of the Canton Bern (EK BE 2017-00501).

For the inclusion in this study, presence of CACLD was a mandatory criterion. CACLD was defined non-invasively according to the Baveno VI criteria, by LSM $\geq 10 \mathrm{kPa}$ using transient elastography (FibroScan, Echosense, France), irrespective of whether it was measured by $\mathrm{M}$ or $\mathrm{XL}$ probe.

We reviewed all consecutive patients who underwent a liver biopsy for diagnostic assessment or staging of a chronic liver disease of any etiology at our center between June 2015 and June 2017. In addition to a LSM $\geq 10 \mathrm{kPa}$, a CAP measurement within 6 months prior to the liver biopsy was required.

We excluded patients with any previous or ongoing hepatic decompensation (defined as ascites, variceal bleeding, hepatic encephalopathy, jaundice), with AST and/or ALT>5 times ULN(19), those with an invalid LSM measurement (IQR/M>0.30), and those in whom the quality of the histological sample was insufficient for a proper interpretation. Figure 1 illustrates the selection flow-chart.

Clinical and laboratory variables were collected at the closest time of liver biopsy (in any case within 3 months). Alcohol consumption at the time of biopsy was classified as none, mild ( $<20 \mathrm{~g} /$ day for women, and $<30 \mathrm{~g} /$ day for men) or moderate/severe (Table 1).

\section{LSM and CAP measurement}

After $\geq 6$ hour fast, LSM and CAP were simultaneously measured with FibroScan 502 Touch (Echosens, Paris, France) using the appropriate probe (M or $\mathrm{XL}$ ), according to the skin-capsule distance. For CAP, attenuation of the transmitted waves was measured automatically in the central frequency of the probe and was expressed in $\mathrm{dB} / \mathrm{m}$. Only patients with valid LSM measurements were included $(9,19)$, and the final LSM (in $\mathrm{kPa}$ ) and CAP values (in $\mathrm{dB} / \mathrm{m}$ ) were given as the median of 10 valid measurements and IQR. LSM was further classified in three categories according to the Baveno VI definitions(6): LSM 10-15 kPa: suggestive of CACLD; LSM >15 kPa: highly suggestive of CACLD; LSM $\geq$ $21 \mathrm{kPa}$ compatible with clinically significant portal hypertension (CSPH). 
The following CAP cut-offs previously published were tested: for any degree of steatosis $\geq 220 \mathrm{~dB} / \mathrm{m}(18)$ and $\geq 248 \mathrm{~dB} / \mathrm{m}(12)$; for S2-S3: $\geq 268 \mathrm{~dB} / \mathrm{m}(12)$; for S3: $\geq 280 \mathrm{~dB} / \mathrm{m}(12)$.

\section{Liver histology}

Liver biopsies were performed using either percutaneous or transjugular access. Briefly, percutaneous liver biopsies were performed with ultrasound assistance, according to Menghini's technique, as previously described(20), using a $17 \mathrm{G}$ biopsy needle (Hepafix®, Braun, Germany). The technique used for transjugular liver biopsy (TJLB) at our center is described in detail elsewhere(21). Samples were fixed in formalin, and were sent to the pathology department. Stainings included Hematoxilin-Eosin, Reticulin and Masson's trichrome (chromotrope aniline blue). An expert pathologist scored fibrosis according to the METAVIR, Brunt, or Ishak scores, as required by the disease etiology. Fibrosis was assessed semiquantitatively, and cirrhosis was defined by the presence of septa and nodules. Advanced chronic liver disease (ACLD) on histology was defined as a bridging fibrosis or cirrhosis, irrespective of the etiology. Steatosis was graded as S0 $(<5 \%)$, S1 $(5-32 \%)$, S2 $(33-66 \%)$, S3 $(>66 \%)$ in relation to the percentage of hepatocytes containing fat droplets(22).

The representativeness of the biopsy was scored using the length of the biopsy specimen and the number of portal tracts. A biopsy was considered optimal in the case of a specimen of at least $20 \mathrm{~mm}$ in length or with at least 15 portal tracts; sufficient when the specimen was not $20 \mathrm{~mm}$ in length, but had at least 11 portal tracts and suboptimal in the remaining cases(23). Patients in whom liver fibrosis could not be staged on histology were excluded from the analysis.

\section{Statistical Analysis}

Quantitative variables normally distributed were expressed as mean and standard deviation, while not-normally distributed variables were shown as median and interquartile range. Comparison of CAP values across to steatosis grade was obtained by Kruskal-Wallis test. Comparison of quantitative variables between two groups was done by using $\mathrm{T}$ test or non-parametric tests, according to the normality of the distribution. Comparison of proportions was done by Fisher Exact test or Kruskal-Wallis test, as appropriate. The discriminative ability of CAP for any grade of steatosis and for S2-S3 
steatosis was studied using the area under the receiving operating characteristic curves (AUROC). Sensitivity, Specificity, positive (+LR) and negative likelihood ratios (-LR) and accuracy were calculated for previously published and pre-defined CAP cut-offs for steatosis(12, 18).

Data were analyzed using SPSS statistics version 23 (SPSS Inc., Chicago, IL, USA).

\section{RESULTS}

461 consecutive patients underwent biopsy (transjugular or percutaneous) for liver disease at our center in the study period. Among them, 147 patients met the Baveno VI definition of CACLD according to LSM. After excluding patients with invalid measurements or exceeding the allowed time period between biopsy and LSM, AST and/or ALT >5 times ULN ( $n=13), 111$ patients (60\% percutaneous biopsy, 40\% TJLB) were included in the final analysis (Figure 1).

The characteristics of the included patients are summarized in Table 1. In brief, 63\% were male, and most were overweight (median BMI $28.1 \mathrm{Kg} / \mathrm{m}^{2}, 36 \%$ obese). More than a half of the population had a metabolic syndrome component: in $32 \%$ the liver disease was due to NAFLD/NASH while an additional $32 \%$ had viral or alcohol related liver disease and metabolic syndrome as a co-factor.

As for transient elastography, $71 \%$ of patients were studied using $M$ probe, while $29 \%$ underwent measurements with XL probe. Median LSM was $16.1 \mathrm{kPa}$ (range 10-75). 42\% of patients had a LSM between 10 and $15 \mathrm{kPa}$, while $58 \%$ had a LSM $\geq 15 \mathrm{kPa}$. In $34 \%$ LSM was $\geq 21 \mathrm{kPa}$.

Table 2 shows the histological stage of fibrosis according to the result of LSM. As shown, using a cut-off of $10 \mathrm{kPa}$, the presence of ACLD was overestimated in about $40 \%$ of patients, while among patients with LSM $\geq 21 \mathrm{kPa} 82 \%$ had ACLD confirmed on histology. As for factors associated with misdiagnosis of CACLD, ongoing alcohol consumption and components of the metabolic syndrome could be identified 
(Supplementary Table 1). Fat content per se or CAP did not explain misclassification of fibrosis in this series.

$87 \%$ of the biopsy samples were large enough to be considered well representative.

On histology, fibrosis was found in $98 / 111$ (88\%), being mild in $11 \%$, moderate in $17 \%$, and bridging fibrosis or cirrhosis in $72 \%$.

CAP and histological steatosis in patients with CACLD diagnosed according to the Baveno VI criteria

In the 111 patients analyzed, the median CAP value was $277 \mathrm{~dB} / \mathrm{m}$. Table 1 shows the distribution of patients according to the different CAP cut-offs. In 78 patients (70\%) CAP was $\geq 220 \mathrm{~dB} / \mathrm{m}$.

On liver biopsy, steatosis was found in $88 / 111$ (79\%) of patients. 44 patients had a high grade steatosis ( $\geq 33 \%$; $\geq S 2$ ). 43 out of these 44 cases were patients with NAFLD/NASH or with alcoholic or viral disease in combination with metabolic syndrome.

CAP was accurate in identifying steatosis of any grade, with an area under the ROC curve (AUROC) of 0.847 (95\%, Cl 0.767-0.926, p <0.0001) (Figure 2, Panel A). Similar good results were found for the detection of S2-S3 steatosis (AUROC 0.860;95\% CI 0.788-0.932, p <0.0001) (Figure 2, Panel B).

Results regarding the accuracy of previously published CAP cut-offs for the detection of any grade of steatosis and high-grade steatosis are shown in Table 3.

To detect any degree of steatosis, accuracy was similar for the two studied cut-offs: $80.2 \%$ for the cut-off $220 \mathrm{~dB} / \mathrm{m}$ (95\% Cl: $71.5 \%-87.1 \%$ ) and $79.3 \%$ for the cut-off 248 $\mathrm{dB} / \mathrm{m}$ (95\% Cl: 70.6\%-86.4\%). However, the threshold of $220 \mathrm{~dB} / \mathrm{m}$ showed a higher sensitivity as compared to the $248 \mathrm{~dB} / \mathrm{m}$ cut-off (85 \% vs. $79 \%$ ). For high-grade (S2-S3) steatosis, the cut-off of $268 \mathrm{~dB} / \mathrm{m}$ showed a very high sensitivity (92.7\%) with moderate specificity (70\%) and acceptable accuracy (78.4\%). For S3 steatosis, the $280 \mathrm{~dB} / \mathrm{m}$ cutoff resulted in a very high sensitivity $(94.1 \%)$ but a poor specificity $(58.5 \%)$ and insufficient accuracy (64\%). 
CAP and histological steatosis in patients with CACLD confirmed on histology

Bridging fibrosis or cirrhosis (CACLD) were diagnosed on histology in 70/111 patients. Within this group, 54 patients $(77 \%)$ had at least some steatosis, and $27(39 \%)$ had a significant or high grade of steatosis (S2-S3). The AUROC of CAP for the diagnosis of any grade of steatosis was $0.804(95 \% \mathrm{Cl} 0.683-0.925 ; \mathrm{p}<0.001)$. For $\mathrm{S} 2-\mathrm{S} 3$ steatosis AUROC was $0.828(95 \% \mathrm{Cl} 0.727-0.930 ; \mathrm{p}<0.0001)$ (Figure 3$)$. In this subgroup of patients with histologically confirmed CACLD, the $220 \mathrm{~dB} / \mathrm{m}$ cut-off resulted in a higher sensitivity and in higher accuracy to detect any degree of steatosis as compared to the $248 \mathrm{~dB} / \mathrm{m}$ cut-off (Table 3). The $268 \mathrm{~dB} / \mathrm{m}$ cut-off had a sensitivity $>90 \%$ and a good accuracy $(81 \%)$ to discriminate between patients with S0-S1 and S2-S3 steatosis. On the other hand, the accuracy of the $280 \mathrm{~dB} / \mathrm{m}$ cut-off to identify patients with S3 steatosis was insufficient (61\%).

Histologically, a diagnosis of cirrhosis was done in 36 cases (27 with any degree of steatosis, of whom 16 had S2-S3 steatosis). The AUROC of CAP to diagnose any steatosis was 0.871 (0.789-0.953) in patients without cirrhosis, and 0.780 (0.589-0.970) in patients with cirrhosis ( $p=0.39$ vs. no cirrhosis). The best CAP cut-off to identify any steatosis in cirrhosis was $219 \mathrm{~dB} / \mathrm{m}$ (Sensitivity $85 \%$, Specificity $78 \%$ ).

The AUROC for S2-S3 steatosis was $0.899(0.824-974)$ in patients without cirrhosis vs. 0.794 (0.637-0.951) in patients with cirrhosis ( $p=0.21$ vs. no cirrhosis). The best CAP cutoff to identify S2-S3 steatosis in cirrhosis was $270 \mathrm{~dB} / \mathrm{m}$ (Sensitivity $87 \%$, Specificity $75 \%)$.

CAP accuracy for steatosis in patients with and without metabolic syndrome As expected, the proportion of any steatosis and S2-S3 steatosis differed between patients with and without metabolic syndrome: any steatosis: $98 \%$ vs. $41 \%$ ( $p=0.03$ ); S2S3 steatosis $59 \%$ vs. $11 \%(p=0.0004)$. The AUROC of CAP to identify any steatosis in patients without metabolic syndrome was good $(0.777$; $95 \% \mathrm{Cl} 0.635-0.919)$ and for S2S3 steatosis, CAP performed similarly in patients with and without metabolic syndrome (AUROC 0.790; 95\% 0.661-0.919 vs. 0.795 ; $95 \% \mathrm{Cl} 0.582-1.000$; comparison of the AUROCS: $p=0.97$ ). As for S2-S3 steatosis, the best cut-off in patients with metabolic syndrome was $303 \mathrm{~dB} / \mathrm{m}$ (Sens $81 \%$, Spec $72 \%$ ), while it appeared lower in patients without metabolic syndrome: $272 \mathrm{~dB} / \mathrm{m}$ (Sens $80 \%$, Spec $82 \%$ ). 
Performance of CAP for steatosis in CACLD according to CAP IQR

The discriminative ability of CAP for any grade of steatosis and for S2-S3 steatosis was similar in patients with CAP IQR $\geq$ or $<40 \mathrm{~dB} / \mathrm{m}$. For any grade of steatosis: AUROC 0.848 (95\% Cl: 0.732-0.964, p<0.001) with CAP IQR $\geq 40$ dB/m vs 0.811 (95\% Cl: 0.6700.951, $p=0.01$ ) in patients with CAP IQR $<40 \mathrm{~dB} / \mathrm{m})$. For S2-S3 steatosis: AUROC 0.917 (95\% Cl 0.834- 0.999, p<0.001) for CAP IQR $\geq 40$ dB/m vs. 0.809 (95\% Cl: 0.693-0.923, $\mathrm{p}<0.001$ ) for CAP $<40 \mathrm{~dB} / \mathrm{m}$. The results did not change when the analysis was restricted to patients with histologically confirmed cACLD (Supplementary Material).

Discriminative ability of CAP for the detection of any steatosis and high-grade steatosis according to $\mathrm{M}$ and $\mathrm{XL}$ probe

In the study population, $\mathrm{M}$ probe was used in $71 \%$ of cases. No major differences in the performance of CAP for steatosis were observed in M vs. XL probe measurements. For the diagnosis of any grade of steatosis, CAP measured by M probe showed an AUROC of $0.795(95 \% \mathrm{Cl} 0.691-0.898 ; \mathrm{p}<0.0001)$. For S2-S3 steatosis, the AUROC was 0.836 (95\% Cl 0.737-0.936; p<0.0001).

Using XL probe, CAP showed an AUROC of 0.958 (95\% Cl 0.884-1.00; $p=0.032)$ for the diagnosis of any grade of steatosis, and of $0.871(95 \% \mathrm{Cl} 0.742-1.00 ; p<0.0001)$ for $\mathrm{S} 2-$ S3 steatosis.

Restricting the analysis to patients with histologically confirmed CACLD, similar results were observed (Supplementary Material). 


\section{DISCUSSION}

The non-invasive identification and risk stratification of patients with CACLD is challenging, but of high importance given that at this stage of the disease the risk of developing portal hypertension and hepatocellular carcinoma is markedly increased. Steatosis is associated with progression of liver fibrosis and to increased risk of clinical decompensation in this population(8). On the other hand, severe steatosis is associated with an overestimation of liver fibrosis by transient elastography(13-15). Hence, reliable tests mirroring the presence and grade of steatosis are needed for a correct risk stratification in the setting of compensated patients assumed or confirmed to have a CACLD.

In the present work, we aimed to assess the reliability of CAP in a contemporary population of patients with CACLD of any etiology diagnosed by LSM according to the Baveno VI criteria(6), using histology as a gold standard for steatosis.

Contrarily to what was previously assumed $(16,17)$, here we were able to prove that steatosis is nowadays very frequent in patients with histologically confirmed bridging fibrosis or cirrhosis, being observed in $77 \%$ of the patients. However, not surprisingly, a significant or severe liver fat content was almost exclusively observed in patients with metabolic syndrome, either as the sole cause of CACLD or as a cofactor added to other etiologies. This implies, that given its negative prognostic role in this population, highgrade steatosis should be clinically suspected and carefully investigated for in patients with CACLD and overweight/obesity and/or metabolic risk factors(8).

In this cohort, we have shown that CAP is able to identify any grade of steatosis and S2S3 steatosis with good accuracy, with results that do not differ from those obtained in patients with less severe chronic liver disease(12). Importantly, the results did not change if we restricted the analysis to patients in whom severe fibrosis or cirrhosis was confirmed on histology.

In our population, a CAP IQR of less than $40 \mathrm{~dB} / \mathrm{m}$ did not further improved CAP diagnostic accuracy, and this differs from the results obtained in milder chronic liver disease(13). 
As for probe size, CAP measured either with $\mathrm{M}$ or $\mathrm{XL}$ probe seemed to be similarly for steatosis or high-grade steatosis, confirming data recently obtained in Asian patients(24). Our study is the first analyzing the diagnostic performance of CAP vs. histology in the setting of CACLD, and our data support that the cut-off of $268 \mathrm{~dB} / \mathrm{m}(12)$ is accurate to rule-out high-grade steatosis in CACLD.

In addition, our results validate the cut-off of $220 \mathrm{~dB} / \mathrm{m}$, which we previously used and that was associated with increased morbidity in patients with CACLD independent of LSM(18). This CAP cut-off holds a $>80 \%$ sensitivity for steatosis detection, performing better than that proposed in the recent meta-analysis for this specific aim(12).

We acknowledge that our study suffers from limitations. It is a retrospective and single center study, and it included a relatively low number of individuals; in addition, our population showed a high prevalence of NAFLD and metabolic syndrome and the pretest probability of steatosis and S2-S3 steatosis was therefore high in our patients.Given to that liver biopsy is an imperfect gold-standard and sampling error can lead to under- or overestimation of fibrosis(25), we cannot exclude that some of the patients considered as misclassified by LSM might instead have CACLD not evident on the biopsy sample. Finally, the Baveno criteria for CACLD identification showed a high proportion of false positive results in the present series; overestimation of fibrosis was more frequent in patients with metabolic syndrome and/or NAFLD/NASH, confirming previous data(4)(15)(16), and suggesting caution in the interpretation of LSM in this context. Importantly, the results regarding CAP accuracy were confirmed in patients with histologically proven bridging fibrosis or cirrhosis.

In conclusion, steatosis was very frequent in a contemporary population of patients with histologically confirmed advanced fibrosis or cirrhosis and severe steatosis likelihood is high in patients with clinical features of metabolic syndrome, irrespective of the main etiology of cACLD. CAP reflected intrahepatic fat content with good accuracy, being better at ruling-out steatosis and high-grade steatosis than at diagnosing them. 
Table 1. Clinical characteristics of the included population $(n=111)$.

\begin{tabular}{|c|c|}
\hline Age, mean \pm SD & $55.3 \pm 12.7$ \\
\hline Male sex, $\mathrm{N}(\%)$ & $70(63)$ \\
\hline Body mass Index $\left(\mathrm{Kg} / \mathrm{m}^{2}\right)$ mean $\pm \mathrm{SD}$ & $28.1 \pm 5.5$ \\
\hline \multicolumn{2}{|l|}{ Etiology } \\
\hline NAFLD/NASH, \% & 32 \\
\hline Any other etiology + metabolic component, \% & 32 \\
\hline viral, \% & 15 \\
\hline alcohol,\% & 4 \\
\hline autoimmune, $\%$ & 6 \\
\hline others, $\%$ & 11 \\
\hline \multicolumn{2}{|l|}{ Laboratory values } \\
\hline Platelets (G/L), mean \pm SD & $185 \pm 93$ \\
\hline Total bilirubin $(\mu \mathrm{mol} / \mathrm{l})$, mean $\pm \mathrm{SD}$ & $19.5 \pm 36.7$ \\
\hline AST (U/I), mean \pm SD & $99 \pm 34$ \\
\hline $\mathrm{ALT}(\mathrm{U} / \mathrm{l})$, mean $\pm \mathrm{SD}$ & $66 \pm 40$ \\
\hline Albumin $(\mathrm{g} / \mathrm{l})$, mean $\pm \mathrm{SD}$ & $37 \pm 10$ \\
\hline Glucose $(\mathrm{mmol} / \mathrm{l})$ mean \pm SD & $6.2 \pm 2.7$ \\
\hline Insulin $(\mathrm{mU} / \mathrm{l})$, mean $\pm \mathrm{SD}$ & $19.8 \pm 14.5$ \\
\hline Total cholesterol $(\mathrm{mmol} / \mathrm{l})$, mean $\pm \mathrm{SD}$ & $4.76 \pm 1.3$ \\
\hline Triglycerides $(\mathrm{mmol} / \mathrm{l})$, mean $\pm \mathrm{SD}$ & $1.6 \pm 1.06$ \\
\hline $\operatorname{ALP}(\mathrm{U} / \mathrm{I})$, mean $\pm \mathrm{SD}$ & $120 \pm 136$ \\
\hline GammaGT (U/I), mean \pm SD & $270 \pm 418$ \\
\hline Creatinine $(\mu \mathrm{mol} / \mathrm{l})$, mean $\pm \mathrm{SD}$ & $75 \pm 32$ \\
\hline \multicolumn{2}{|l|}{ Transient elastography probe used } \\
\hline M Probe (\%) & 71 \\
\hline XL Probe $(\%)$ & 29 \\
\hline \multicolumn{2}{|l|}{ Liver stiffness } \\
\hline LS: $10-15 \mathrm{kPa}, \mathrm{N}(\%)$ & $47(42.3)$ \\
\hline LS: >15 kPa, N (\%) & $64(57.7)$ \\
\hline
\end{tabular}




\begin{tabular}{|l|c|}
\hline LS: $\geq 21 \mathrm{kPa}, \mathrm{N}(\%)$ & $34(30.6)$ \\
\hline CAP & $78(70.3)$ \\
\hline$\geq 220 \mathrm{~dB} / \mathrm{m}, \mathrm{N}(\%)$ & $69(62.2)$ \\
\hline$\geq 248 \mathrm{~dB} / \mathrm{m}, \mathrm{N}(\%)$ & $59(53.2)$ \\
\hline$\geq 268 \mathrm{~dB} / \mathrm{m}, \mathrm{N}(\%)$ & $55(49.5)$ \\
\hline$\geq 280 \mathrm{~dB} / \mathrm{m}, \mathrm{N}(\%)$ & $69(62)$ \\
\hline CAP IQR <40, N (\%) & \\
\hline Representativeness of the biopsy & 48 \\
\hline Optimal, \% & 39 \\
\hline Sufficient, \% & 13 \\
\hline Suboptimal,\% & $23(18.5)$ \\
\hline Steatosis grade & $42(33.9)$ \\
\hline S0 (<5\%), N (\%) & $24(19.3)$ \\
\hline S1 (5-32\%), N (\%) & $20(16.2)$ \\
\hline S2 (33-66\%), N (\%) & \\
\hline S3 (>66\%), N (\%) & $41(36.9)$ \\
\hline Fibrosis on LB & $70(63.1)$ \\
\hline F0-F2, N (\%) & \\
\hline F3-F4, N (\%) & \\
\hline
\end{tabular}




\begin{tabular}{|c|c|c|c|}
\hline & $\begin{array}{c}\text { No fibrosis or } \\
\text { mild fibrosis }\end{array}$ & $\begin{array}{c}\text { Significant fibrosis, no } \\
\text { bridging fibrosis no } \\
\text { cirrhosis }\end{array}$ & $\begin{array}{c}\text { Bridging fibrosis or } \\
\text { cirrhosis (cACLD) }\end{array}$ \\
\hline $\begin{array}{c}\text { LSM 10-15 kPa (cACLD } \\
\text { likely), } \mathbf{n = 4 7}\end{array}$ & $10(21 \%)$ & $10(21 \%)$ & $27(58 \%)$ \\
\hline LSM $\geq \mathbf{1 5} \mathbf{~ k P a}$ & $14(22 \%)$ & $7(11 \%)$ & $43(67 \%)$ \\
(cACLD very likely), $\mathbf{n = 6 4}$ & & & $28(82 \%)$ \\
\hline $\begin{array}{c}\text { Subgroup with LSM } \\
\geq 21 \mathrm{kPa}\end{array}$ & $4(12 \%)$ & & \\
\hline $\begin{array}{c}\text { (clinically significant } \\
\text { portal hypertension) } \mathbf{n = 3 4}\end{array}$ & & & \\
\hline
\end{tabular}

Table 2. Liver fibrosis severity on histology according to the LSM cut-offs proposed by the Baveno VI consensus. 
Table 3. Diagnostic performance of CAP using previously published cut-offs.

\begin{tabular}{|c|c|c|c|c|c|c|}
\hline & Cut-off & $\begin{array}{l}\text { Sensitivity } \\
\%(95 \% \mathrm{Cl})\end{array}$ & $\begin{array}{l}\text { Specificity } \\
\%(95 \% \mathrm{Cl})\end{array}$ & $\begin{array}{c}\text { +LR } \\
(95 \% \mathrm{Cl})\end{array}$ & $\begin{array}{c}\text {-LR } \\
(95 \% \mathrm{Cl})\end{array}$ & $\begin{array}{l}\text { Accuracy } \\
\%(95 \% \mathrm{Cl})\end{array}$ \\
\hline & \multicolumn{6}{|c|}{ Complete study population: cACLD diagnosed by LSM } \\
\hline \multirow[t]{2}{*}{$\begin{array}{c}\text { Any } \\
\text { steatosis }\end{array}$} & $220 \mathrm{~dB} / \mathrm{m}$ & $\begin{array}{c}85.0 \\
(75.3-92.0)\end{array}$ & $\begin{array}{c}67.7 \\
(48.6-83.3)\end{array}$ & $\begin{array}{c}2.63 \\
(1.57-4.42)\end{array}$ & $\begin{array}{c}0.22 \\
(0.12-0.39)\end{array}$ & $\begin{array}{c}80.2 \\
(71.5-87.1)\end{array}$ \\
\hline & $248 \mathrm{~dB} / \mathrm{m}$ & $\begin{array}{c}78.7 \\
(68.2-87.1)\end{array}$ & $\begin{array}{c}80.6 \\
(62.5-92.6)\end{array}$ & $\begin{array}{c}4.07 \\
(1.97-8.42)\end{array}$ & $\begin{array}{c}0.26 \\
(0.17-0.42)\end{array}$ & $\begin{array}{c}79.3 \\
(70.5-86.4)\end{array}$ \\
\hline $\begin{array}{c}\text { S1-S0 vs. } \\
\text { S2-S3 }\end{array}$ & $268 \mathrm{~dB} / \mathrm{m}$ & $\begin{array}{c}92.7 \\
(80.1-98.5)\end{array}$ & $\begin{array}{c}70.0 \\
(57.9-80.4)\end{array}$ & $\begin{array}{c}3.09 \\
(2.14-4.46)\end{array}$ & $\begin{array}{c}0.10 \\
(0.03-0.31)\end{array}$ & $\begin{array}{c}78.4 \\
(69.6-85.6)\end{array}$ \\
\hline S3 & $280 \mathrm{~dB} / \mathrm{m}$ & $\begin{array}{c}94.1 \\
(71.3-99.9)\end{array}$ & $\begin{array}{c}58.5 \\
(47.9-68.6)\end{array}$ & $\begin{array}{c}2.27 \\
(1.74-2.97)\end{array}$ & $\begin{array}{c}0.10 \\
(0.01-0.68)\end{array}$ & $\begin{array}{c}64.0 \\
(54.3-72.9)\end{array}$ \\
\hline & \multicolumn{6}{|c|}{ Histologically confirmed cACLD } \\
\hline $\begin{array}{c}\text { Any } \\
\text { steatosis }\end{array}$ & $220 \mathrm{~dB} / \mathrm{m}$ & $\begin{array}{c}83.3 \\
(70.7-92.1)\end{array}$ & $\begin{array}{c}62.5 \\
(35.4-84.8)\end{array}$ & $\begin{array}{c}2.22 \\
(1.17-4.23)\end{array}$ & $\begin{array}{c}0.27 \\
(0.13-0.54)\end{array}$ & $\begin{array}{c}78.6 \\
(67.1-87.5)\end{array}$ \\
\hline & $248 \mathrm{~dB} / \mathrm{m}$ & $\begin{array}{c}74.1 \\
(60.4-85.0)\end{array}$ & $\begin{array}{c}81.2 \\
(54.4-96.0)\end{array}$ & $\begin{array}{c}3.95 \\
(1.41-11.1)\end{array}$ & $\begin{array}{c}0.32 \\
(0.19-0.53)\end{array}$ & $\begin{array}{c}75.7 \\
(64.0-85.2)\end{array}$ \\
\hline $\begin{array}{c}\text { S1-S0 vs. } \\
\text { S2-S3 }\end{array}$ & $268 \mathrm{~dB} / \mathrm{m}$ & $\begin{array}{c}92.6 \\
(75.7-99.1)\end{array}$ & $\begin{array}{c}74.4 \\
(58.8-86.5)\end{array}$ & $\begin{array}{c}3.62 \\
(2.15-6.09)\end{array}$ & $\begin{array}{c}0.10 \\
(0.03-0.38)\end{array}$ & $\begin{array}{c}81.4 \\
(70.3-89.7)\end{array}$ \\
\hline S3 & $280 \mathrm{~dB} / \mathrm{m}$ & $\begin{array}{c}87.5 \\
(47.4-99.7)\end{array}$ & $\begin{array}{c}58.1 \\
(44.9-70.5)\end{array}$ & $\begin{array}{c}2.09 \\
(1.41-3.09)\end{array}$ & $\begin{array}{c}0.22 \\
(0.03-1.36)\end{array}$ & $\begin{array}{c}61.4 \\
(49.0-72.8)\end{array}$ \\
\hline
\end{tabular}


Figure Legends

Figure 1. Flow-chart of the study population. LS, liver stiffness; CAP, controlled attenuation parameter; cACLD, compensated advanced chronic liver disease.

Figure 2. Discriminative ability of CAP to diagnose steatosis in patients diagnosed of CACLD using the Baveno VI criteria (LSM $\geq 10 \mathrm{kPa}$ ). Panel A. CAP for any grade of steatosis. AUROC 0.847 (95\% Cl 0.767-0.926, p<0.0001). Panel B. CAP for S2-S3 steatosis. AUROC $0.860(95 \% \mathrm{Cl} 0.788-0.932, \mathrm{p}<0.0001)$.

Figure 3. Discriminative ability of CAP for S2-S3 steatosis in patients with histologically proven bridging fibrosis or cirrhosis. AUROC 0.828 (95\% Cl $0.727-$ 0.930, $p<0.0001)$. 


\section{References}

1. Younossi ZM, Koenig AB, Abdelatif D, et al. Global epidemiology of nonalcoholic fatty liver disease-Meta-analytic assessment of prevalence, incidence, and outcomes. Hepatology 2016;64:73-84.

2. Singh S, Allen AM, Wang Z, et al. Fibrosis progression in nonalcoholic fatty liver vs nonalcoholic steatohepatitis: a systematic review and meta-analysis of paired-biopsy studies. Clin Gastroenterol Hepatol 2015;13:643-654.

3. Dyson J, Jaques B, Chattopadyhay D, et al. Hepatocellular cancer: the impact of obesity, type 2 diabetes and a multidisciplinary team. J Hepatol 2014;60:110-117.

4. Leandro G, Mangia A, Hui J, et al. Relationship between steatosis, inflammation, and fibrosis in chronic hepatitis C: a meta-analysis of individual patient data.

Gastroenterology 2006;130:1636-1642.

5. Terrault NA, Bzowej NH, Chang KM, et al. AASLD guidelines for treatment of chronic hepatitis B. Hepatology 2016;63:261-283.

6. de Franchis R, Baveno VIFaculty. Expanding consensus in portal hypertension: Report of the Baveno VI Consensus Workshop: Stratifying risk and individualizing care for portal hypertension. J Hepatol 2015;63:743-752.

7. Berzigotti A, Garcia-Tsao G, Bosch J, et al. Obesity is an independent risk factor for clinical decompensation in patients with cirrhosis. Hepatology 2011;54:555-561. 8. Everhart JE, Lok AS, Kim HY, et al. Weight-related effects on disease progression in the hepatitis $\mathrm{C}$ antiviral long-term treatment against cirrhosis trial. Gastroenterology 2009;137:549-557.

9. European Association for Study of Liver, Asociacion Latinoamericana para el Estudio del Higado. EASL-ALEH Clinical Practice Guidelines: Non-invasive tests for evaluation of liver disease severity and prognosis. J Hepatol 2015;63:237-264.

10. Berzigotti A. Getting closer to a point-of-care diagnostic assessment in patients with chronic liver disease: controlled attenuation parameter for steatosis. J Hepatol 2014;60:910-912.

11. Berzigotti A, Ferraioli G, Bota $S$, et al. Novel ultrasound-based methods to assess liver disease: The game has just begun. Dig Liver Dis 2018;50:107-112. 
12. Karlas T, Petroff D, Sasso M, et al. Individual patient data meta-analysis of controlled attenuation parameter (CAP) technology for assessing steatosis. J Hepatol 2017;66:1022-1030.

13. Wong VW, Petta S, Hiriart JB, et al. Validity criteria for the diagnosis of fatty liver by M probe-based controlled attenuation parameter. J Hepatol 2017;67:577-584.

14. Petta S, Wong VW, Camma C, et al. Improved noninvasive prediction of liver fibrosis by liver stiffness measurement in patients with nonalcoholic fatty liver disease accounting for controlled attenuation parameter values. Hepatology 2017;65:1145-1155. 15. Karlas T, Petroff D, Sasso M, et al. Impact of controlled attenuation parameter on detecting fibrosis using liver stiffness measurement. Aliment Pharmacol Ther 2018;47:989-1000.

16. van der Poorten D, Samer CF, Ramezani-Moghadam M, et al. Hepatic fat loss in advanced nonalcoholic steatohepatitis: are alterations in serum adiponectin the cause? Hepatology 2013;57:2180-2188.

17. Lok AS, Everhart JE, Chung RT, et al. Evolution of hepatic steatosis in patients with advanced hepatitis $\mathrm{C}$ : results from the hepatitis $\mathrm{C}$ antiviral long-term treatment against cirrhosis (HALT-C) trial. Hepatology 2009;49:1828-1837.

18. Margini C, Stirnimann G, De Gottardi A, et al. Prognostic Significance of Controlled Attenuation Parameter in Patients With Compensated Advanced Chronic Liver Disease. Hepatol Commun. 2018 Jul 24;2:929-940.

19. Dietrich CF, Bamber J, Berzigotti A, et al. EFSUMB Guidelines and Recommendations on the Clinical Use of Liver Ultrasound Elastography, Update 2017. Ultraschall Med 2017;38:e16-e47.

20. Gilmore IT, Burroughs A, Murray-Lyon IM, et al. Indications, methods, and outcomes of percutaneous liver biopsy in England and Wales: an audit by the British Society of Gastroenterology and the Royal College of Physicians of London. Gut 1995;36:437-441.

21. Hari A, Nair HK, De Gottardi A, et al. Diagnostic hepatic haemodynamic techniques: safety and radiation exposure. Liver Int 2017;37:148-154.

22. Kleiner DE, Brunt EM, Van Natta M, et al. Design and validation of a histological scoring system for nonalcoholic fatty liver disease. Hepatology 2005;41:1313-1321. 
23. Fryer $E$, Wang LM, Verrill C, et al. How often do our liver core biopsies reach current definitions of adequacy? J Clin Pathol 2013;66:1087-1089.

24. Chan WK, Nik Mustapha NR, Wong GL, et al. Controlled attenuation parameter using the FibroScan $(R) X L$ probe for quantification of hepatic steatosis for non-alcoholic fatty liver disease in an Asian population. United European Gastroenterol J 2017;5:76-85. 25. Colloredo G, Guido M, Sonzogni A, et al. Impact of liver biopsy size on histological evaluation of chronic viral hepatitis: the smaller the sample, the milder the disease. $J$ Hepatol 2003;39:239-244. 


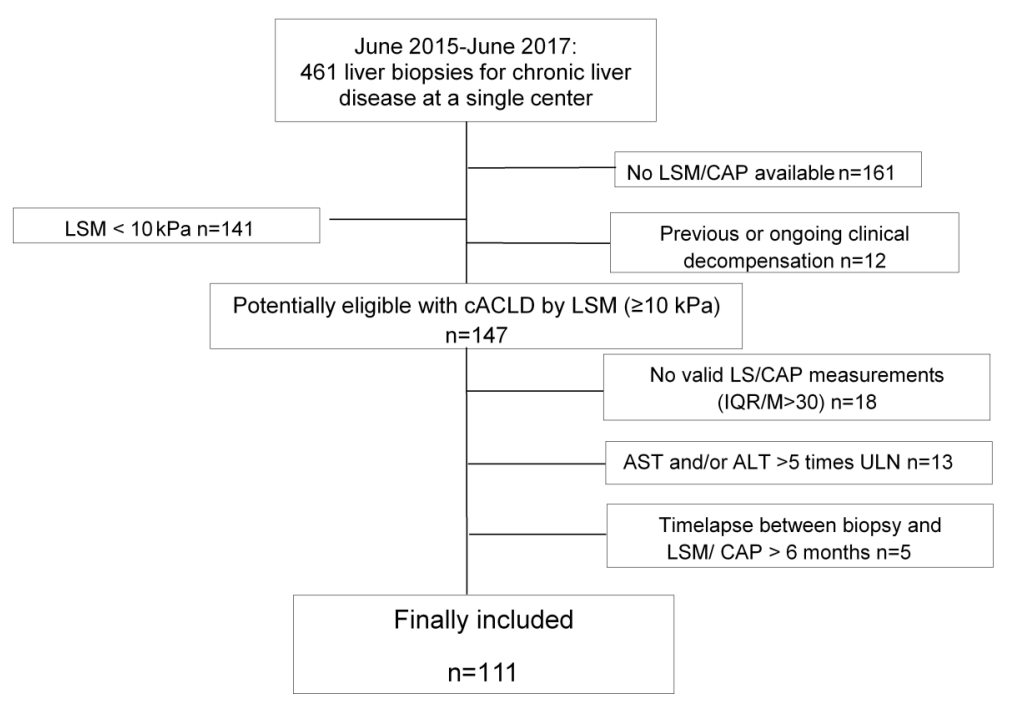

liv_14325_f1.tif

This article is protected by copyright. All rights reserved 

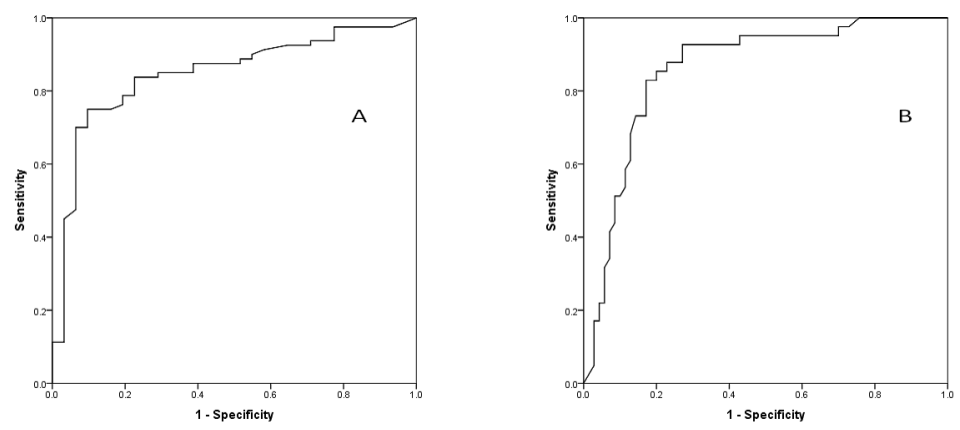

liv_14325_f2.tif 

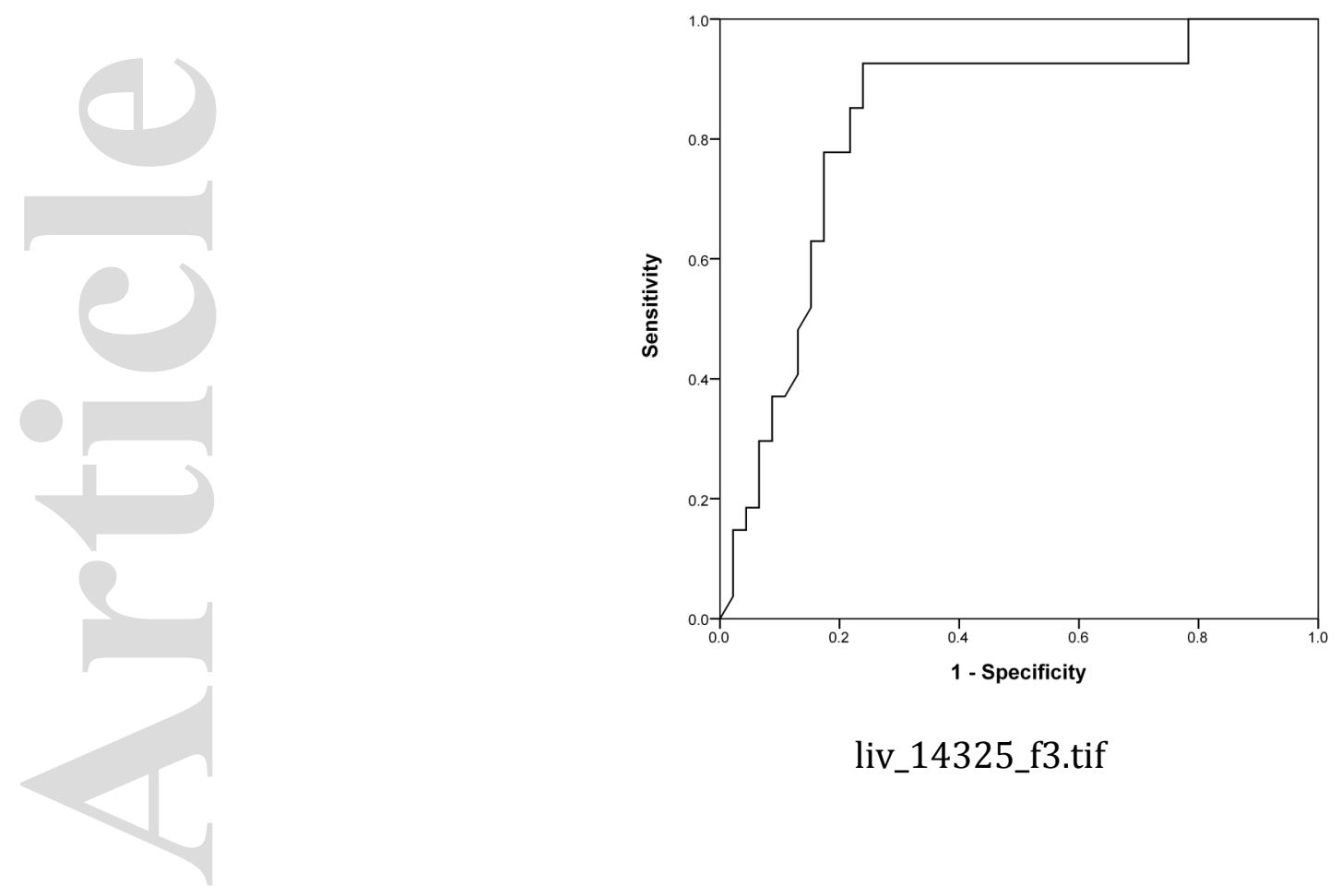

liv_14325_f3.tif 